Pierre PoILeCoT ${ }^{1}$

Nema-Soua LouA ${ }^{2}$

${ }^{1}$ Cirad

Département Environnements

et sociétés

Unité de recherche Animal

et gestion intégrée des risques

TA C/22 E

Campus international de Baillarguet

34398 Montpellier Cedex 5

France

$2 \mathrm{Smfg} /$ Mont Nimba

BP 2046, Conakry

République de Guinée

\title{
Les feux dans les savanes des monts Nimba, Guinée
}

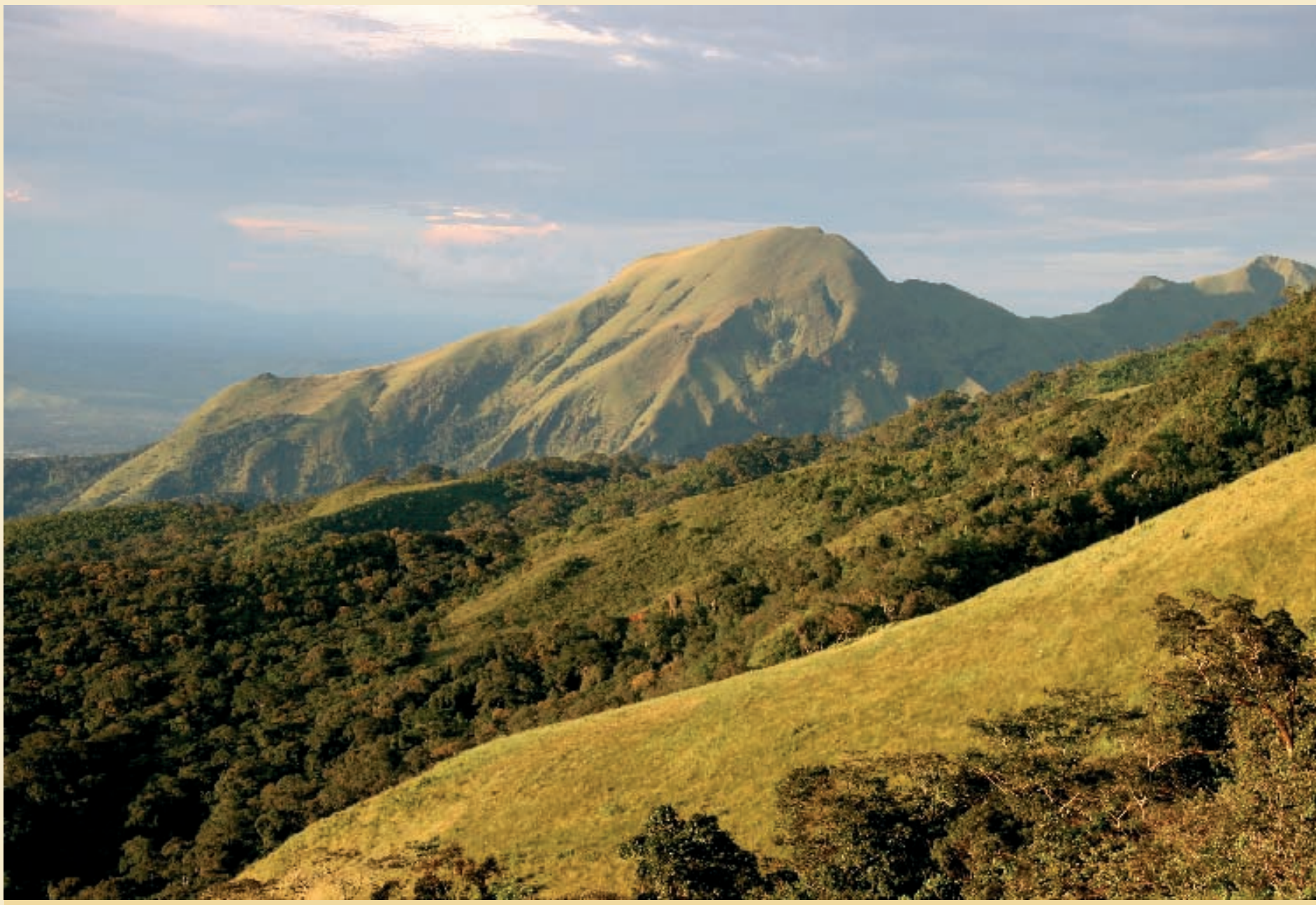

Photo 1.

Mosaïque forêt-savane dans la partie nord de la chaîne des monts Nimba.

Photo P. Poilecot. 


\section{RÉSUMÉ}

\section{LES FEUX DANS LES SAVANES DES MONTS NIMBA, GUINÉE}

Le massif des monts Nimba, au sudest de la République de Guinée, fut érigé en réserve de la biosphère et en site du patrimoine mondial en 1981 dans un but de conservation d'un écosystème d'une richesse biologique extraordinaire. Le développement d'un projet d'exploitation minière est en cours d'étude, ce qui a incité les autorités à demander une étude d’impact environnemental de façon à évaluer les risques de dégradation du milieu naturel dans le cas d'une mise en exploitation du fer dans un futur proche. L'étude était composée d'un inventaire rapide de la biodiversité et d'une expertise sur l'impact des feux de brousse dans les savanes qui fut réalisée en mars 2008. Les feux de brousse sont provoqués dans le cadre d'activités pastorales, agricoles ou de braconnage. Dans les savanes de basse altitude, les feux annuels permettent le maintien en équilibre et la diversité floristique des formations herbacées et évitent leur embroussaillement. Les feux sont moins fréquents dans les savanes submontagnardes et n'ont pas d'impact négatif sur le tapis herbacé mais altèrent les lisières et le sous-bois des forêts de ravin. La pratique des feux précoces est la seule qui permette de concilier production de pâtures et conservation dans cet écosystème de savane, sans avoir d'impact négatif sur la végétation et la faune terrestre. Elle limite, voire empêche, les feux tardifs dommageables à la végétation et aux sols. Les feux de brousse ne doivent pas être considérés comme un fléau pour le massif des monts Nimba si l'on tient compte de l'origine secondaire des savanes et du façonnage par l'homme des phytocénoses actuelles par une succession d'activités agricoles et pastorales.

Mots-clés : savane, feu de brousse, mont Nimba, Guinée.

\section{ABSTRACT}

\section{BUSH FIRES IN THE SAVANNA LANDS OF MOUNT NIMBA, GUINEA}

The Nimba Range, in the south-east of the Republic of Guinea, was listed as a Biosphere Reserve and World Heritage Site in 1981 in the interests of conserving and ecosystem of outstanding biological wealth. The development of a mining project is now under investigation, which has prompted the authorities to request an environmental impact study in order to assess the risks of degradation of the natural environment should iron ore extraction begin in the near future. The study involved a brief biodiversity inventory and an expert assessment of the impact of bush fires in savannas in March 2008. Bush fires occur as a result of pastoral, agricultural or poaching activities. In low altitude savannas, yearly bush fires help to maintain the balance of flora perversity in herbaceous formations and to prevent woodland encroachment. Bush fires are less frequent in sub-montane savanna and while they do not adversely affect herbaceous ground cover, they will modify woodland edges and undergrowth in forests and ravines. Early season fires are the only way of reconciling plaster management and conservation in the savanna ecosystem without negative impacts for the terrestrial vegetation and fauna. They live it and even prevent late season fires that damage vegetation and soils. Bush fires should not be regarded as a scourge in the Nimba Range given the secondary origin of its savanna lands and the way humans have shaped the plant communities that exist today through a succession of agricultural and pastoral activities.

Keywords: savanna, bush fires, Mount Nimba, Guinea.

\section{RESUMEN}

\section{LOS FUEGOS EN LAS SABANAS DE LOS MONTES NIMBA, GUINEA}

El macizo de los montes Nimba, al sudeste de la república de Guinea, fue declarado Reserva de la Biosfera y Sitio del Patrimonio Mundial en 1981 con el objetivo de proteger un ecosistema de una extraordinaria riqueza biológica. El desarrollo de un proyecto de explotación minera está en fase de estudio, esto ha llevado a las autoridades a solicitar un estudio de impacto ambiental para evaluar los riesgos de degradación del entorno natural en el caso de una próxima explotación del hierro. Dicho estudio se compuso de un rápido inventario de la biodiversidad y de un informe pericial sobre el impacto de los fuegos de matorral en las sabanas que fue realizado en marzo de 2008. Los fuegos de matorral se provocan en el marco de actividades pastoriles, agrícolas o de furtivismo. En las sabanas de tierras bajas, los fuegos anuales permiten mantener el equilibrio y la diversidad florística de las formaciones herbáceas y evitan su enmalezamiento. Los fuegos son menos frecuentes en las sabanas submontanas y no tienen impacto negativo sobre el estrato herbáceo, pero alteran los linderos y el sotobosque de los bosques de cañadas. La práctica de fuegos precoces es la única que permite compaginar la producción de pastizales y la conservación en este ecosistema de sabana, sin tener impactos negativos sobre la vegetación y la fauna terrestre. Dicha práctica limita, e incluso impide, los incendios tardíos, perjudiciales para la vegetación y los suelos. Los fuegos de matorral no deben considerarse como una plaga para el macizo de los montes Nimba si se tiene en cuenta el origen secundario de las sabanas y el modelado antrópico de las fitocenosis actuales por una sucesión de actividades agrícolas y pastoriles.

Palabras clave: sabanas, fuego de matorral, Montes Nimba, Guinea. 


\section{Introduction}

Les monts Nimba, culminant à $1752 \mathrm{~m}$, constituent l'un des massifs montagneux les plus élevés de l'Afrique de l'Ouest. Cette chaîne montagneuse à cheval sur la Guinée, le Liberia et la Côte d'Ivoire, très riche en minerai de fer, a attiré l'attention de nombreux scientifiques depuis 1942 jusqu'en 1956-57. En 1944, au vu de la richesse biologique du massif, fut créée la réserve naturelle intégrale des monts Nimba englobant les parties guinéenne et ivoirienne. Celle-ci fut ensuite érigée en réserve de la biosphère en 1980 et en site du patrimoine mondial en 1981, à l'initiative du programme Mab (Man and Biosphere) de l'Unesco. La grande diversité de la végétation du Nimba, des forêts ombrophiles et mésophiles des régions inférieures aux forêts à Parinari excelsa et aux savanes d'altitude, conduit à une diversité également très importante des peuplements animaux. En effet, près de sept cents espèces de vertébrés et deux mille cinq cents d'invertébrés (l'identification de nouvelles espèces étant toujours en cours) ont été décrites dans ce massif. Le Nimba abrite également quatre cents espèces d'oiseaux, soixante-neuf de reptiles et soixante-six d'amphibiens.

L'originalité des savanes ou « prairies » d'altitude réside dans l'individualisation d'une faune orophile renfermant de nombreuses espèces présumées endémiques. La flore par contre est pauvre en endémiques propres au Nimba, du fait de son altitude peu importante, mais il existe un endémisme montagnard ouest-africain, au travers des différents massifs du Nimba, du massif du Fon-Simandou (Guinée) et du massif du Loma (Sierra Leone), caractérisant la région.

Malgré les travaux de recherche considérables effectués dans les monts Nimba sur la géomorphologie, le climat, la végétation, la flore et la faune, la situation demeure critique du fait d'un manque de surveillance du massif et des pressions anthropiques de plus en plus fortes exer-
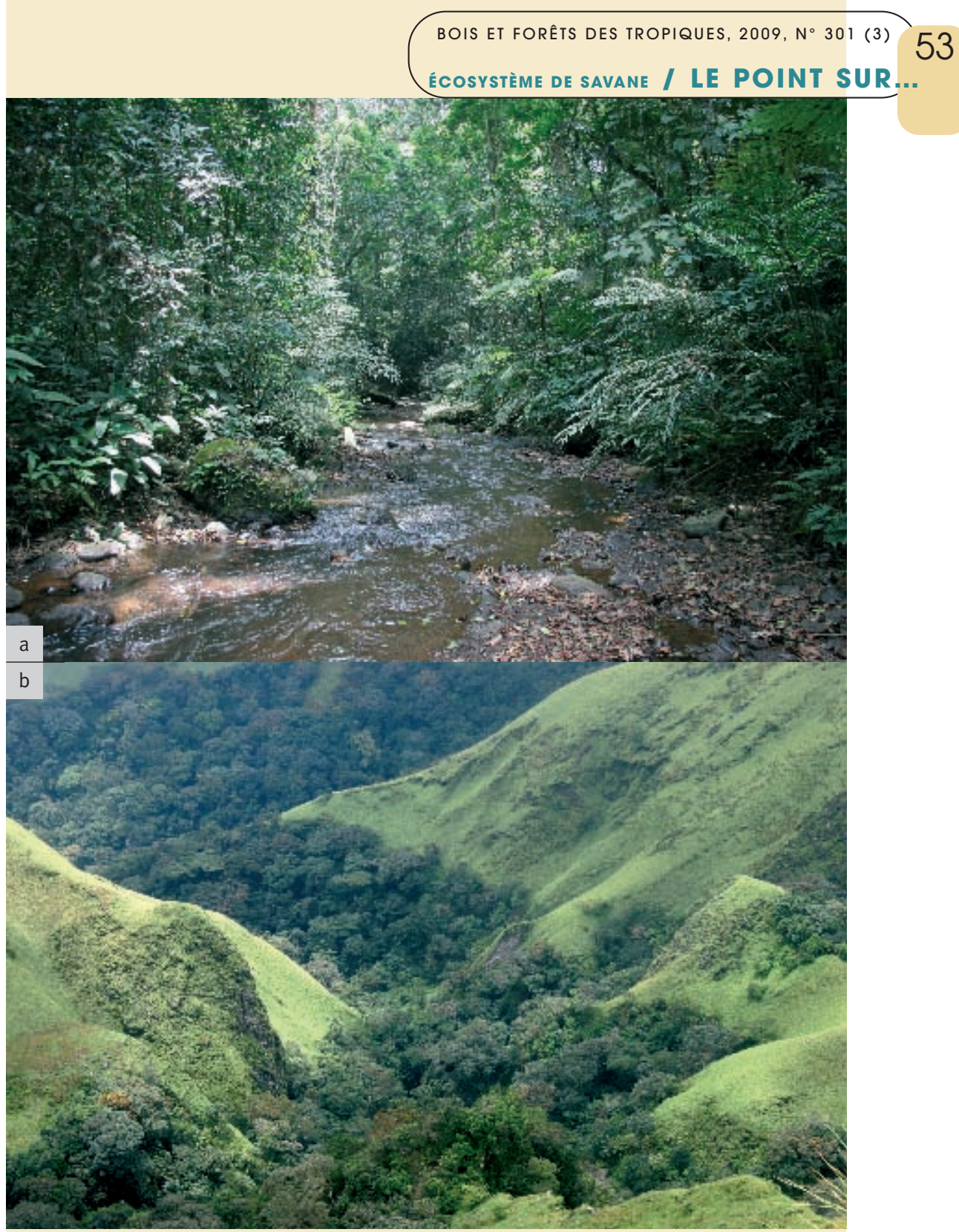

Photos 2.

a. Forêt dense humide de piémont ;

b. Forêt montagnarde à Parinari excelsa s'élevant jusqu'aux crêtes des sommets en suivant les ravins plus ou moins encaissés.

Photo P. Poilecot.

cées sur le massif et sa périphérie. C'est dans ce contexte qu'une étude d'impact environnemental d'un projet d'exploitation minière a été demandée par les autorités. En parallèle d'un inventaire biologique rapide (Biopa - Biodiversity Preliminary Assessment), effectué par l'association Afrique Nature International (Afnat), fut réalisée une expertise sur les feux de brousse pour lesquels peu de données étaient disponibles (POILECOT, 2008). En novembre 2008, une étude sur la végétation herbacée des savanes du massif a permis d'approfondir les connaissances sur la composition floristique et la structure du tapis herbacé (PoIlecot et al., 2008).

En Guinée, la réserve de la biosphère des monts Nimba couvre 145200 ha. Le site du patrimoine mondial, incluant une réserve intégrale, s'étend sur 12500 ha. Un périmètre minier de 1550 ha a été alloué aux activités minières, dans lequel la Société des minerais de fer de Guinée (Smfg) étudie le potentiel minier au sein d'une concession de 625 ha. 


\section{Le Nimba: diversité géographique et biologique}

\section{Modelé et climat}

Au sud-est de la dorsale guinéenne (qui s'étend sur près de $500 \mathrm{~km}$ des grès du massif guinéen du Fouta-Djalon à la région de Man en Côte d'Ivoire), le massif des monts Nimba, situé entre $7^{\circ} 25^{\prime}$ et $7^{\circ} 45^{\prime}$ de latitude Nord et entre $8^{\circ} 20$ et $8^{\circ} 35^{\prime}$ de longitude Ouest, marque les frontières entre la Côte d'Ivoire, la Guinée et le Liberia et s'élève brusquement de plus de $1000 \mathrm{~m}$ au-dessus du piémont environnant (figure 1).

La région septentrionale du massif, appartenant à la Guinée, porte le point culminant formé par le mont Richard-Molard à 1752 m, et constitue un "pivot » avec la partie ivoirienne. À l'extrême nord-est, la chaîne se divise en trois parties à partir d'un plateau situé à $1600 \mathrm{~m}$ d'altitude. En dessous de 700-800 m, elle repose sur le socle antécambrien formé de roches cristallines et métamorphiques. La ligne des crêtes s'appuie sur des masses d'itabirites (quartzites à minerai de fer très durs), plus résistantes que le socle cristallin (Lamotte, Rougerie, 1998). La vigueur du relief, à l'origine de climats locaux très variés, et la complexité de la structure géologique ont déterminé la coexistence de multiples formations végétales (photo 1).

La chaîne des monts Nimba est située au confluent de trois domaines climatiques régionaux (LAM OTTE, 1998) mais elle est caractérisée globalement par un climat subéquatorial montagnard avec une saison sèche qui s'étend de novembre à avril. La pluviosité annuelle, sur le versant guinéen, est très variable d'une année à l'autre et du sud au nord. Elle est estimée à environ $1500 \mathrm{~mm}$ au niveau du piémont (500 m d'altitude) mais peut atteindre $3000 \mathrm{~mm}$ au niveau des crêtes. En altitude, la

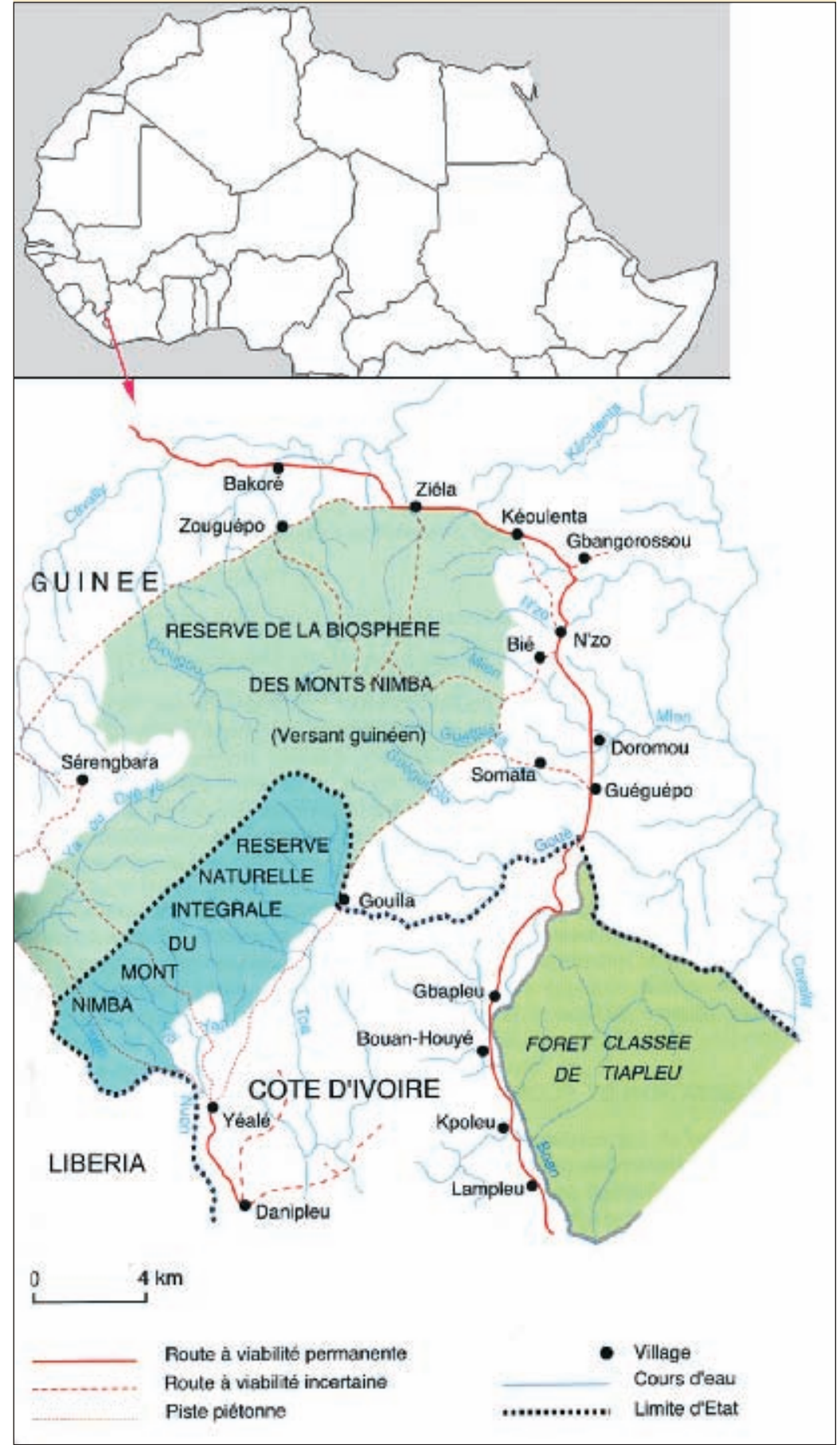

Figure 1.

Localisation du massif des monts Nimba en Afrique de l'Ouest.

température moyenne varie entre 16 et $21^{\circ} \mathrm{C}$ alors qu'elle se situe entre 22 et $27^{\circ} \mathrm{C}$ au niveau du piémont. Les pentes nord et est sont davantage en contact avec l'harmattan en provenance du désert, et donc plus sèches que celles exposées au sud et à l'ouest qui sont soumises aux effets de la mousson. 


\section{Une vaste mosaïque forêts-savanes}

Sur le versant guinéen, les forêts de l'étage guinéo-équatorial inférieur, dans le sud-ouest, correspondent à des forêts ombrophiles caractérisées par l'association à Tarrieta utilis, Lophira alata et Mapania spp. (SCHNELL, 1952). Elles s'opposent aux forêts mésophiles qui occupent les plateaux et les pentes du Nimba nord-est (photo 2 a). Les régions basses sont occupées par une association à Triplochiton scleroxylon et Chrysophyllum perpulchrum. Les forêts submontagnardes des pentes sont en partie secondarisées du fait de défrichements mais leur composition est très proche des précédentes. Les formations forestières de l'étage guinéo-équatorial supérieur, à partir de 1000 m d'altitude, s’individualisent par la présence de Parinari excelsa, en particulier dans les ravins supérieurs (photo $2 \mathrm{~b}$ ).

\section{Savane de piémont et "prairies " montagnardes}

Au pied du versant sud-est du Nimba, de vastes savanes caractérisent la zone de piémont à environ 500-550 m d'altitude (région de Gouéla) (photo 3).

Ces formations, composant un damier de clairières et de forêts denses, reposent sur des cuirasses ferrugineuses compactes subhorizontales. Leur composition dépend de la profondeur du sol et elles sont représentées par :

- des savanes à couvert arboré relativement clair comprenant, entre autres, Terminalia schimperiana, Ficus glumosa, Bridelia ferruginea et Syzygium guineense dominant un tapis graminéen composé de Andropogon macrophyllus, Hyparrhenia diplandra, Anadelphia leptocoma, Rhytachne rottboellioides;

- des savanes basses, sur la cuirasse affleurante, caractérisées par la robuste graminée Loudetia arundinacea avec également d'autres Poaceae comme Rhytachne rottboellioides, et des touradons d'Afrotrilepis pilosa (Cyperaceae) ;

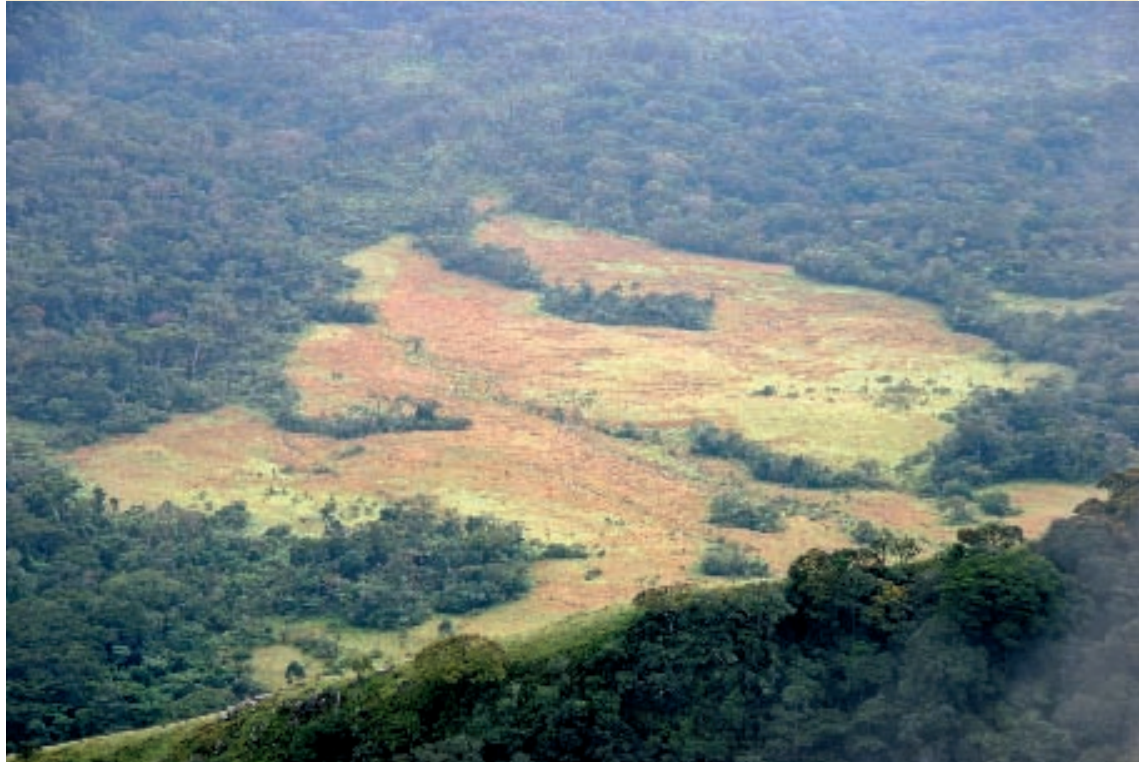

Photo 3.

Savane de piémont sur le versant nord-est de la chaîne des monts Nimba.

Photo P. Poilecot.

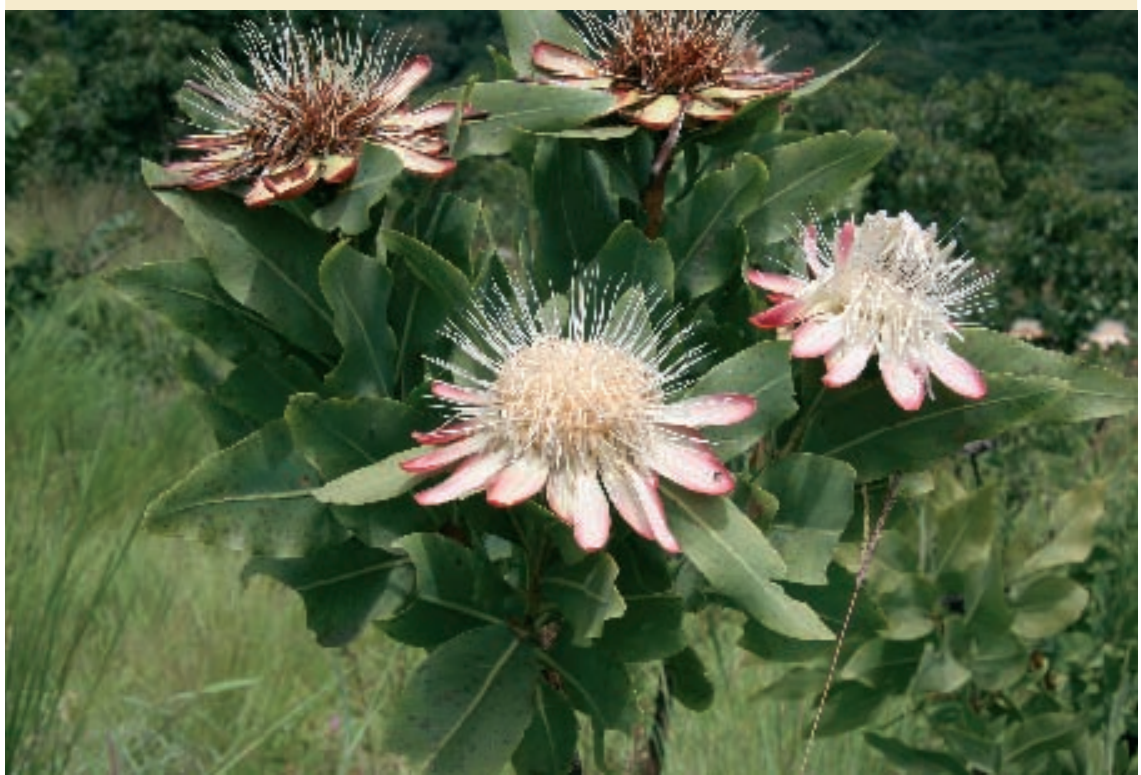

Photo 4.

Protea madiensis subsp. occidentalis.

Photo P. Poilecot.

- des marécages sur la cuirasse affleurante comprenant des espèces plus hygrophiles comme Genlisea africana, Drosera indica, Eriocaulon pumilum et Xyris straminea.

Les savanes submontagnardes, à partir de 800-900 m, occupent des sols généralement minces recouvrant une roche ferrugineuse, très dure et souvent plissée. Le peuplement ligneux, très ouvert, est constitué d'espèces communes aux savanes de piémont auxquelles est associé un arbuste grégaire, Protea madiensis subsp. occidentalis, qui disparaît ensuite aux plus hautes altitudes (photo 4). 

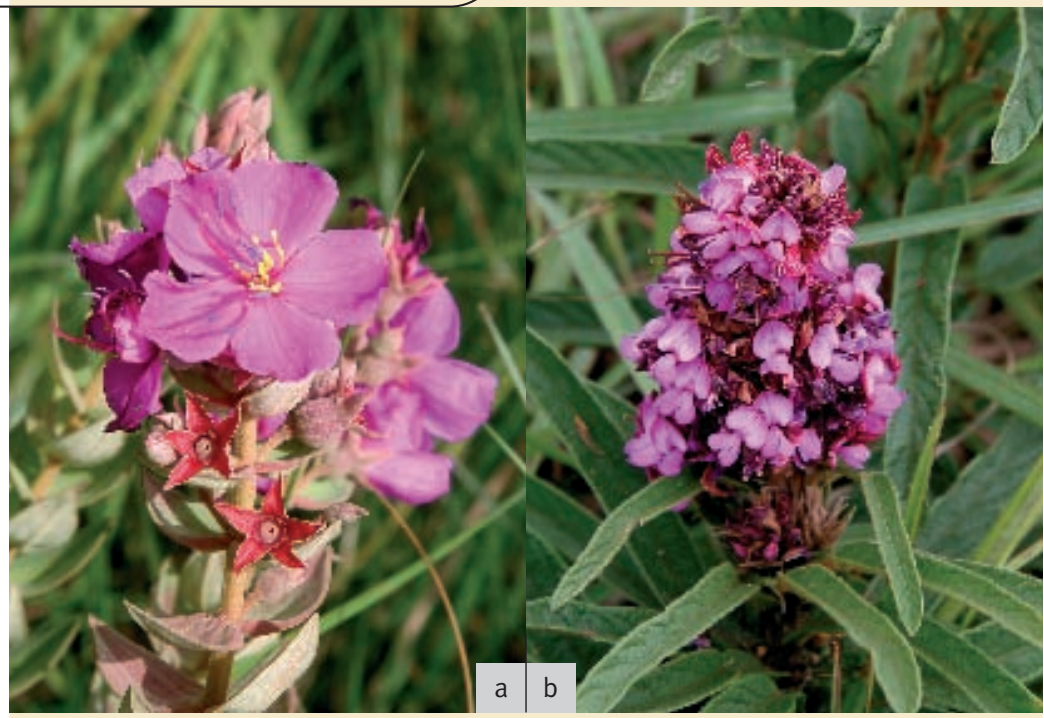

Photos 5.

a. Heterotis amplexicaulis ; b. Droogmansia scaettaiana.

Photo P. Poilecot.

La strate herbacée est composée de grandes graminées vivaces telles A. schirensis, Hyparrhenia diplandra, H. subplumosa, H. mutica, Monocymbium ceresiiforme, Elymandra androphila et Loudetia kagerensis (SCHNELL, 1987, 1998 ; FOURNIER, 1987 ; POILECOT et al., 2008). Elles sont associées à diverses espèces arbustives comme Kotschya ochreata, Heterotis amplexicaulis (photo 5 a), Droogmansia scaettaiana (photo 5 b) ou Dolichos tonkouiensis.

Ce type de savane, remontant sur les pentes jusqu'à 1300 m d'altitude, est ensuite dominé par Loudetia kagerensis, une graminée qui constitue l'essentiel de la biomasse herbacée (photo 6 a). Une petite légumineuse à fleurs jaunes, Eriosema parviflorum susbp. parviflorum, lui est souvent associée (photo 6 b).

Des zones de cuirasse affleurante modifient localement la composition du tapis herbacé. Elles sont caractérisées par une strate graminéenne renfermant Loudetia simplex, Andropogon africanus, $A$. perligulatus, Ctenium newtonii, Anadelphia afzeliana, A. leptocoma, Rhytachne rottboellioides, Panicum griffonii et $P$. congoense associées à diverses Cyperaceae (photo 7).

Les étages supérieurs renferment un certain nombre d'orophytes, localisés aux niveaux les plus élevés et installés sur des substrats rocheux ou rocailleux, comme Helichrysum mechowianum, Dolichos nimbaensis et Blaeria spicata subsp. mannii. Deux espèces seulement, rupicoles,
Osbeckia porteresii et Asplenium schnellii, peuvent être considérées comme endémiques du Nimba.

La transition forêts-savane, sur les pentes inférieures, est très souvent assurée par un peuplement dense pratiquement monospécifique d'Andropogon macrophyllus (photo 8).

\section{Les feux de brousse sur le versant guinéen des monts Nimba}

En Guinée, comme dans la plupart des cas en Afrique subsaharienne, les feux de brousse sont le plus souvent d'origine anthropique (volontaire ou accidentelle) et dus aux éleveurs (pour provoquer une repousse d'herbe), agriculteurs (feux culturaux non contrôlés qui débordent dans les savanes adjacentes; photo 9), braconniers, collecteurs de miel, villageois (faciliter les déplacements dans les savanes, éliminer les parasites, maintenir la production de pailles destinées aux toitures, favoriser la floraison des arbres fruitiers, pratiquer des feux rituels).

Bien que les causes naturelles soient réelles (foudre, chute de pierres provoquant des étincelles), elles ne peuvent être à l'origine de feux parcourant annuellement de grandes superficies de savanes. Les incendies et leur régularité sont davantage caractéristiques d'une volonté humaine.

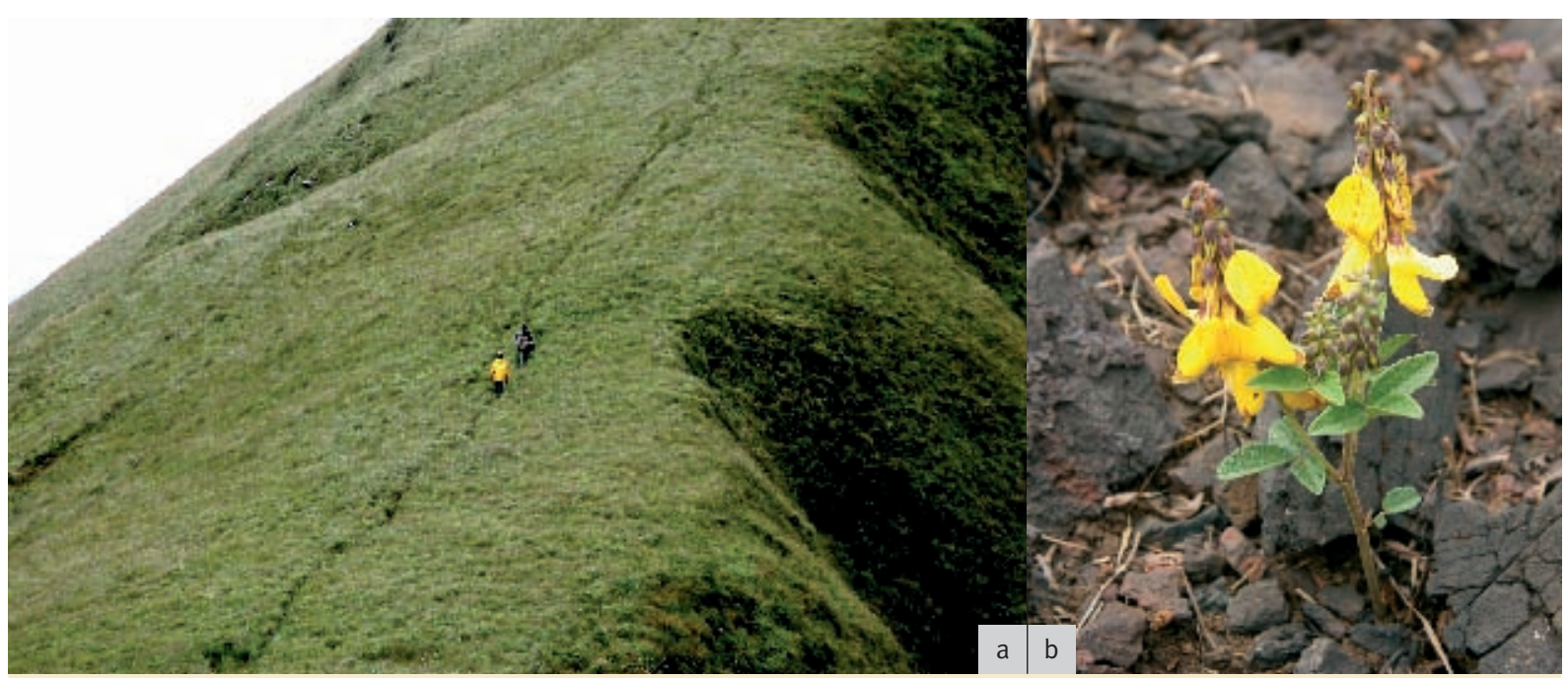

Photos 6.

a. Savane montagnarde à Loudetia kagerensis, sur une pente forte à $1500 \mathrm{~m}$ d'altitude :

b. Eriosema parviflorum subsp. parviflorum en fleur après le passage du feu.

Photo P. Poilecot. 
Dans les savanes des monts Nimba, les feux commencent à partir du mois de novembre.

Il est fréquent de remarquer sur le piémont ouest, localement, des bosquets de ligneux constitués d'espèces pionnières comme Harungana madagascariensis, Trema orientalis, Gaertnera paniculata, Albizia zygia, Alchornea cordifolia et Macaranga hurifolia, caractéristiques des stades de la régénération de la forêt dense mésophile. Cette évolution témoigne d'un rythme de feux irrégulier et de l'impact de feux précoces.

\section{Impacts des feux de brousse sur les milieux naturels ou semi-anthropisés}

Les observations relatives aux feux de brousse sur le versant guinéen des monts Nimba sont localisées sur la figure 2 .

\section{Impact du feu sur le sol}

Dans les savanes, l'approvisionnement du sol en matière organique résulte davantage du système souterrain (racinaire) que du système aérien

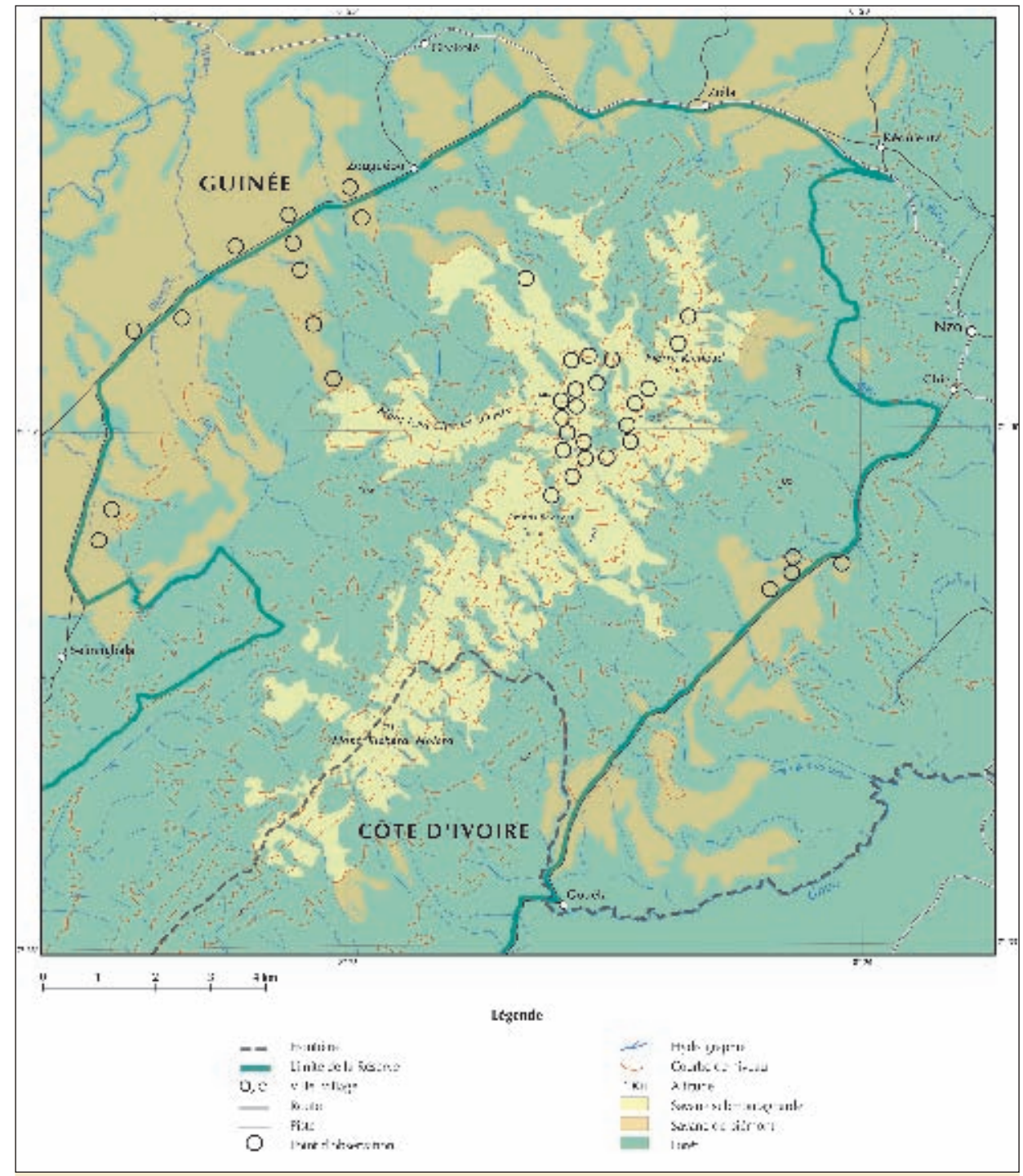

Figure 2.

Carte illustrant la localisation des points d'observation relatifs aux feux de brousse sur le versant guinéen des monts Nimba. (chaumes et feuilles). Une savane produisant $6 \mathrm{t} / \mathrm{ha}$ de matière sèche aérienne libère annuellement, par son système racinaire, entre 6 et $20 \mathrm{t} / \mathrm{ha}$ de matière organique. Le passage $\mathrm{du}$ feu minéralise de façon brutale une énorme quantité de matière organique, estimée de $2-4 \mathrm{t} / \mathrm{h}$ a en zone soudanienne à 6-8 t/ha dans les savanes guinéennes. De plus, il restitue, au travers des cendres, les éléments constitutifs (azote, phosphore et potassium) de la matière végétale. Les apports par la pluie et la fixation biologique (en particulier pour l'azote) peuvent compenser pour une large part les pertes par drainage et combustion. L'impact du feu sur la richesse du sol et sur la matière organique est donc réduit.

Le sol dénudé, après le passage du feu, peut faciliter l'érosion hydrique sur les terrains à forte pente. De rares observations ont permis de conclure, localement, à un impact négatif des feux de brousse sur les sols du Nimba. Les feux précoces permettent une régénération rapide du tapis herbacé qui est bien en place au moment des premières grosses pluies. Les sols d'altitude, rocheux ou rocailleux, constituent une défense naturelle contre l'érosion hydrique et l'absence temporaire de végétation ne représente pas un risque de dégradation.

\section{Impact du feu sur les rivières}

Le régime de la plupart des cours d'eau en savane est saisonnier, avec un débit réduit, voire nul, au cours de la saison sèche mais torrentiel en saison des pluies. Ces rivières subissent l'impact des activités humaines conduites dans le bassin versant où elles sont produites. Bien qu'aucune étude n'ait été menée sur ce sujet dans le Nimba, les conséquences du feu sur le réseau hydrographique peuvent être directes comme un dépôt d'ammoniaque à partir de la fumée ou indirectes du fait d'une disparition brusque de la végétation pouvant entraîner une 
érosion, avec une augmentation des dépôts de sédiments et de matière organique dans les rivières et des perturbations dans les cycles de nutriments. Les incendies peuvent avoir un effet néfaste sur la végétation des galeries forestières, en modifiant leur composition floristique et leur structure ainsi qu'en conduisant à la mort de certains arbres, de même que sur la faune aquatique (DougLAs et al., 2003). Cependant, une comparaison entre un régime de feux précoces et une protection contre le feu sur les bassins versants met en évidence que les premiers n'entraînent aucune dégradation tant en ce qui concerne la qualité de l'eau que la végétation des galeries forestières ou la végétation aquatique.

\section{Impact du feu sur la végétation}

Les feux tardifs, plus violents, ont un impact sur la végétation ligneuse : arbres et arbustes tués, diminution de la surface terrière, régénération supprimée ou retardée, graines détruites, etc. Cependant, la plupart des arbres ont une forte capacité à rejeter à la suite du passage du feu. Les incendies ont une incidence sur la phénologie des ligneux, en réduisant l'intensité de la floraison et/ou de la fructification. Il en découle une production moindre de graines avec un effet réducteur sur le recrutement potentiel de jeunes individus au sein des peuplements. Des effets indirects se manifestent également pour la faune qui utilise le pollen ou qui consomme les fruits et les graines.

Certains auteurs (BowmAn et al., 1995) concluent que la composition floristique et la diversité du tapis graminéen ne varient pas ou très peu sous l'action des feux, quel que soit leur régime. La richesse spécifique est plus forte en cas de feu modéré que dans le cas d'une protection intégrale. YeAton et al. (1986) rapportent un déclin de la diversité de la richesse spécifique du tapis herbacé si l'intervalle entre les feux augmente.

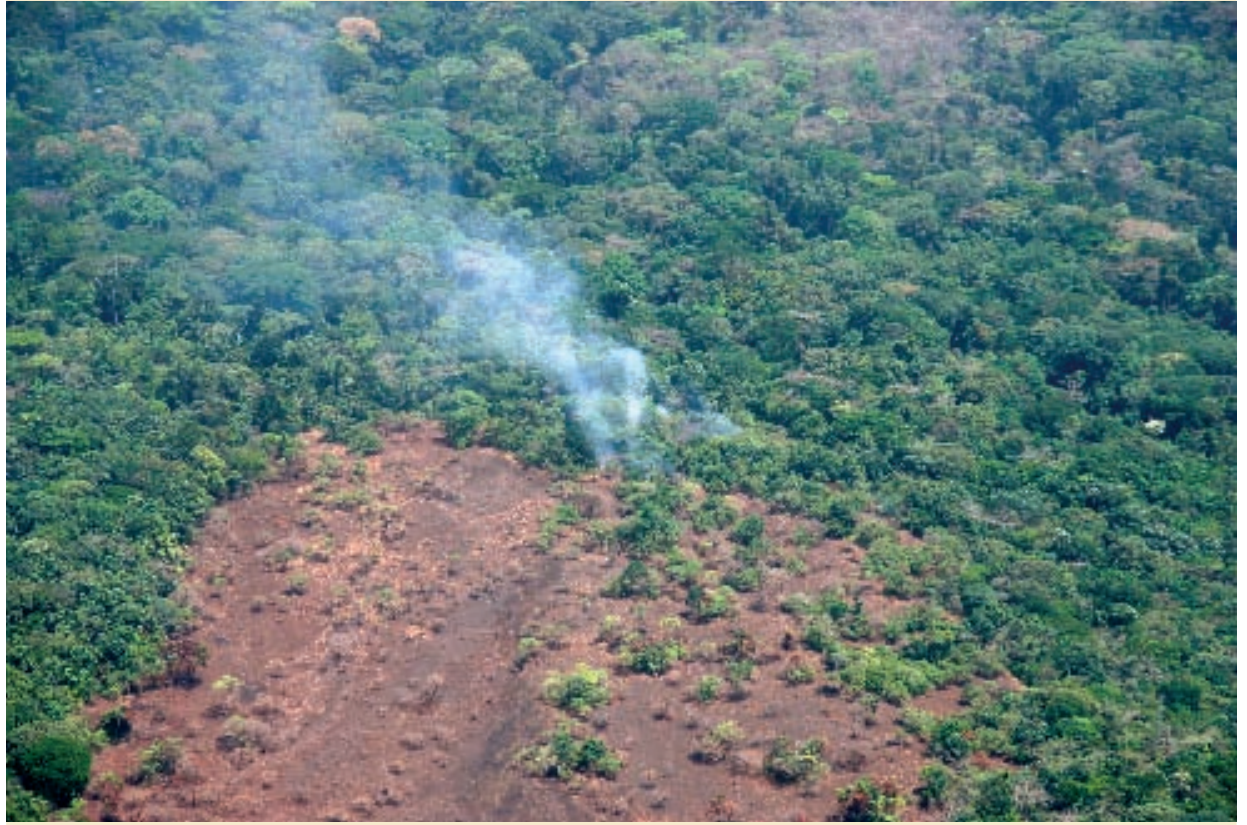

Photo 9.

Feu de braconniers (fumage de viande) s'étant étendu aux savanes de piémont puis à celles des crêtes, à 1600 m d'altitude (mont Pierré-Richaud).

Photo P. Poilecot.

Les savanes de piémont des monts Nimba seraient d'origine secondaire, à la suite de la destruction des forêts mésophiles sur cuirasse n'ayant pu se reconstituer après les défrichements. La carapace, dans ce cas, n'est pas une conséquence de la déforestation mais l'une des causes essentielles de l'instabilité de la forêt et de sa disparition. À la fin février, les savanes de piémont ont généralement subi le passage du feu et sont souvent à l'origine des incendies dans les " prairies » des crêtes. Les feux constituent un facteur d'équilibre de ces savanes sur cuirasse affleurante qui représentent un groupement édaphique assez stable. La suppression des feux dans ces formations de piémont entraînerait une modification de cet équilibre tant du point de vue physionomique, floristique que faunistique. Les lisières forestières, du fait de dépôts de matières organiques, tendent localement à progresser sur la savane à partir d'espèces pionnières des forêts et galeries environnantes (KoeChlin, 1998).
L'origine des « prairies » montagnardes résulterait de la régression récente de la forêt montagnarde sous l'influence humaine. Ces prairies se maintiendraient grâce aux feux de brousse périodiques (SCHNELL, 1952). Les feux naturels, parfois observés dans le Nimba, au rôle secondaire dans le processus de déforestation, sont généralement éteints par la pluie d'orages. Ils ont tendance à se développer en suivant les courants ascendants du vent et ne descendent pas vers les forêts inférieures. Les incendies responsables de la dégradation initiale de la forêt ont certainement été allumés dans les savanes inférieures, par les agriculteurs et les chasseurs, et ont ensuite régulièrement suivi les pentes pour atteindre les crêtes. Le feu aurait éliminé ou réduit la forêt occupant les têtes de ravin, conduisant la végétation des lisières à évoluer vers la savane herbeuse montagnarde. La présence d'orophytes, n'appartenant pas à la flore tropicale des basses altitudes, plaide également pour une origine secondaire de ces savanes. 


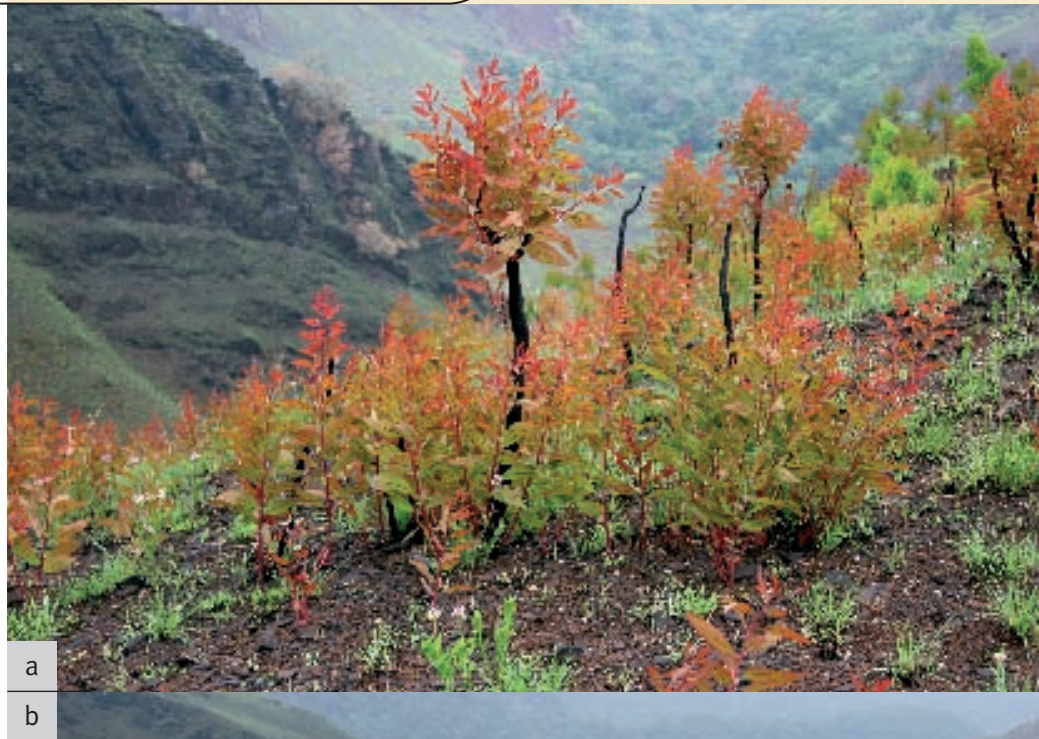

Les feux de brousse ne semblent pas avoir d'impact négatif sur la végétation de ces savanes. Ils interviennent comme un facteur écologique dans leur fonctionnement. Les espèces observées, tant dans les savanes brûlées que dans celles en cours de régénération après le passage des incendies, sont les mêmes que celles décrites par SCHNELL en 1952.

En revanche, l'extension de Melinis minutiflora se poursuit dans les savanes d'altitude. Cette robuste Poaceae vivace, très agressive, souvent décrite comme une plante invasive, est bien d'origine africaine (JACQUES-FÉlIX, 1987). Elle colonise les talus de route, les lisières forestières, le rebord des carapaces latéritiques et pénètre dans les « prairies » d'altitude où elle tend localement à se substituer à Loudetia kagerensis.

Héliophile, sociale, elle étendrait ainsi son aire de distribution sur le Nimba du fait du passage moins fréquent des feux de brousse (photo 11).

Alors que la régénération des espèces herbacées explose après le passage du feu, surtout à la suite des premières averses (février-mars), les savanes non brûlées sont réduites à un tapis herbeux desséché, très fourni, rabattu par la pluie et le vent. Quelques plantes comme Vernonia nimbaensis ou Phyllanthus alpestris émettent de longues tiges feuillées audessus de la strate graminéenne pour fleurir. L'absence de feux et l'excès de matériel mort limitent la floraison de nombreuses espèces au profit de l'activité végétative des plantes. Le couvert graminéen tend à diminuer du fait de l'impossibilité pour les pérennes de taller (occupation horizontale de l'espace) puisque les plantes recherchent la lumière en développant de longs chaumes verticaux. Il s'ensuit une modification de la stratification du tapis herbacé avec une diminution de la production végétale. La suppression des feux de brousse dans les savanes du Nimba pourrait entraîner un appauvrissement de la diversité et de la richesse floristiques, comme le souligne ARBONNIER (2008) pour les savanes d'altitude d'Afrique centrale. 
L'action du feu est semblable à celle, mécanique, d'une coupe. Les incendies conservent le cortège floristique de la savane, maintiennent les Poaceae productives et éliminent ou freinent le développement des plantes annuelles, rudérales et des espèces forestières. La repousse n'est pas induite par le feu (il la rend plus visible) mais elle dépend de la rétention en eau du sol : le reverdissement se produit en savane non brûlée, mais de façon moins intense, si l'humidité contenue dans le sol est suffisante.

Ces particularités sont importantes à prendre en considération pour la gestion des pâturages, surtout dans les savanes de piémont. Sous l'effet du pâturage, une seule génération de ligneux (arbres et arbustes) a tendance à dominer, avec une surpopulation de jeunes individus. Un pâturage intense épuise les graminées pérennes et la biomasse végétale combustible diminue, si bien que le feu devient moins agressif au profit des ligneux, avec un risque d'embroussaillement du milieu.

L'impact des feux de brousse sur le Nimba est certainement le plus visible dans les formations forestières, tant dans la zone de piémont qu'en altitude. Aux forêts de basse et moyenne altitudes se sont substituées des formations secondaires (forêts reconstituées, fourrés arbustifs, jachères), caractérisées par l'abondance du palmier à l'huile (Elaeis guineensis) maintenu et disséminé par l'homme. Les lisières des forêts mésophiles et des galeries sont peu affectées par les incendies de savanes de piémont : quelques arbustes sont plus ou moins touchés par les flammes mais leur survie n'est pas menacée.

La dégradation des forêts d'altitude, plus inquiétante, peut être attribuée à deux facteurs : les feux de brousse et le ravinement (dû à des causes naturelles : vent, éboulements de roches). L'impact du feu est partout apparent sur le contact forêt-savane dans les ravins d'altitude, où la végétation est souvent affectée par les flammes, avec pénétration du feu dans le sous-bois des forêts qui cause des

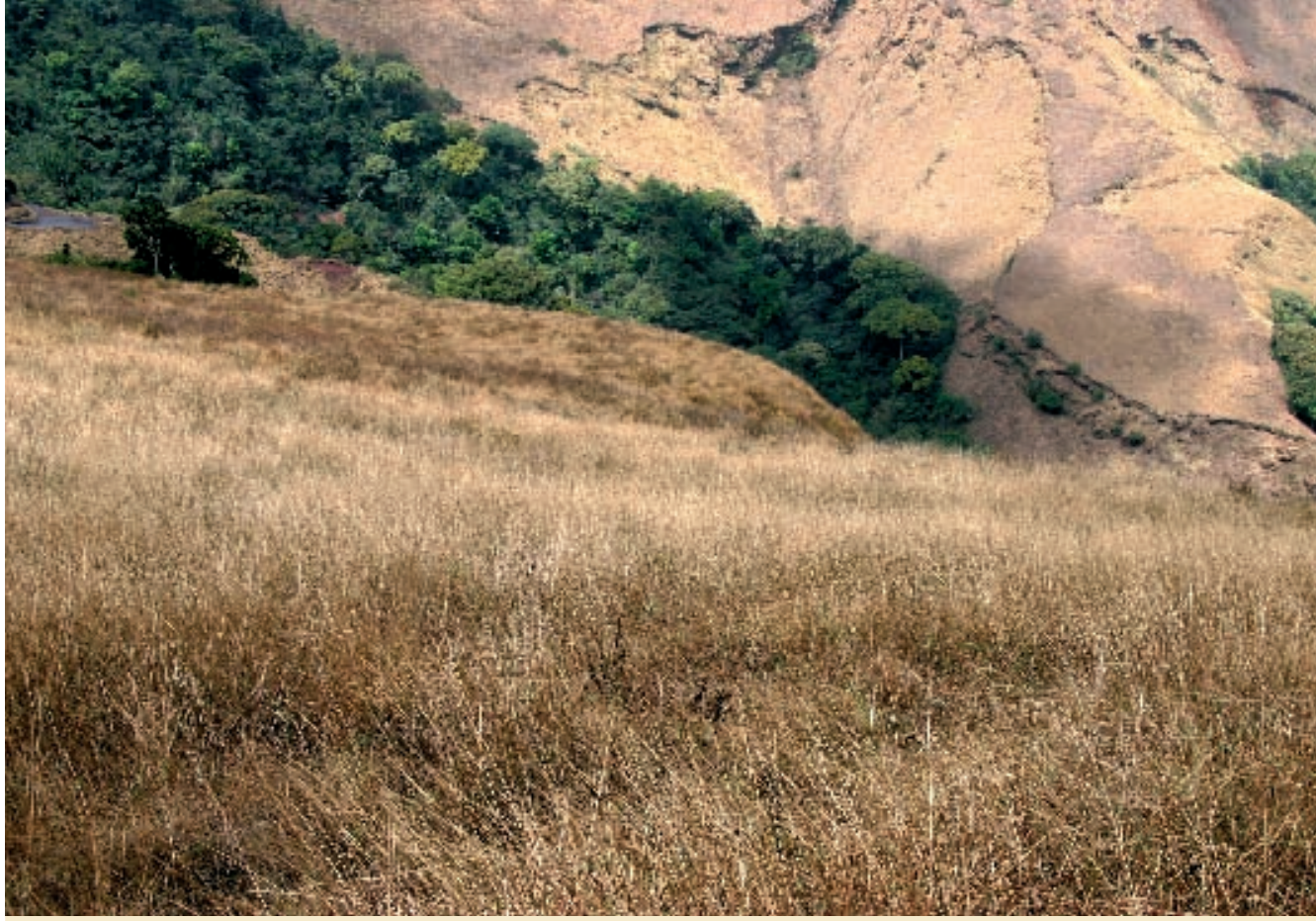

Photo 11.

Envahissement de la savane d'altitude à Loudetia kagerensis par Melinis minutiflora.

Photo P. Poilecot.

dommages aux arbres (photos 12 a et b). Des rudérales comme Mikania scandens et Rubus fellatae, dans les lisières forestières, sont les témoins d'une dégradation des formations d'altitude. Malgré cela, ces espèces ne constituent qu'une perturbation transitoire, sur des sites localisés, du fait des conditions édaphiques contraignantes pour elles. De petits Parinari excelsa existent parfois dans ces zones de transition, principalement sur les terrasses rocheuses surplombant les ravins indiquant une régression de la forêt.

La suppression des feux serait, sans aucun doute, favorable aux formations forestières montagnardes.

Par contre, la recolonisation de ces savanes par la forêt basse à Parinari excelsa semble peu probable. En effet, comme le souligne SCHNELL (1952), cette formation se serait installée jadis dans des conditions écologiques plus favorables et elle est peu dynamique quant à sa reconstitution.

\section{Impact du feu sur la faune}

L'originalité des savanes d'altitude a conduit les scientifiques à étudier en détail la faune des monts Nimba. Dans l'état des connaissances actuelles, il semble difficile de pouvoir définir un endémisme propre au Nimba du fait de lacunes concernant les autres massifs de l'Afrique au sud du Sahara (LAMotTE, Roy, 2003). Certaines espèces comme le micropotamogale (Micropotamogale lamottei), endémique de la dorsale guinéenne, et le crapaud vivipare Nectophrynoides occidentalis (photo 13), très commun dans les savanes d'altitude du Nimba, ont largement contribué à la réputation de ce massif (LAUGINIE, 2008). 


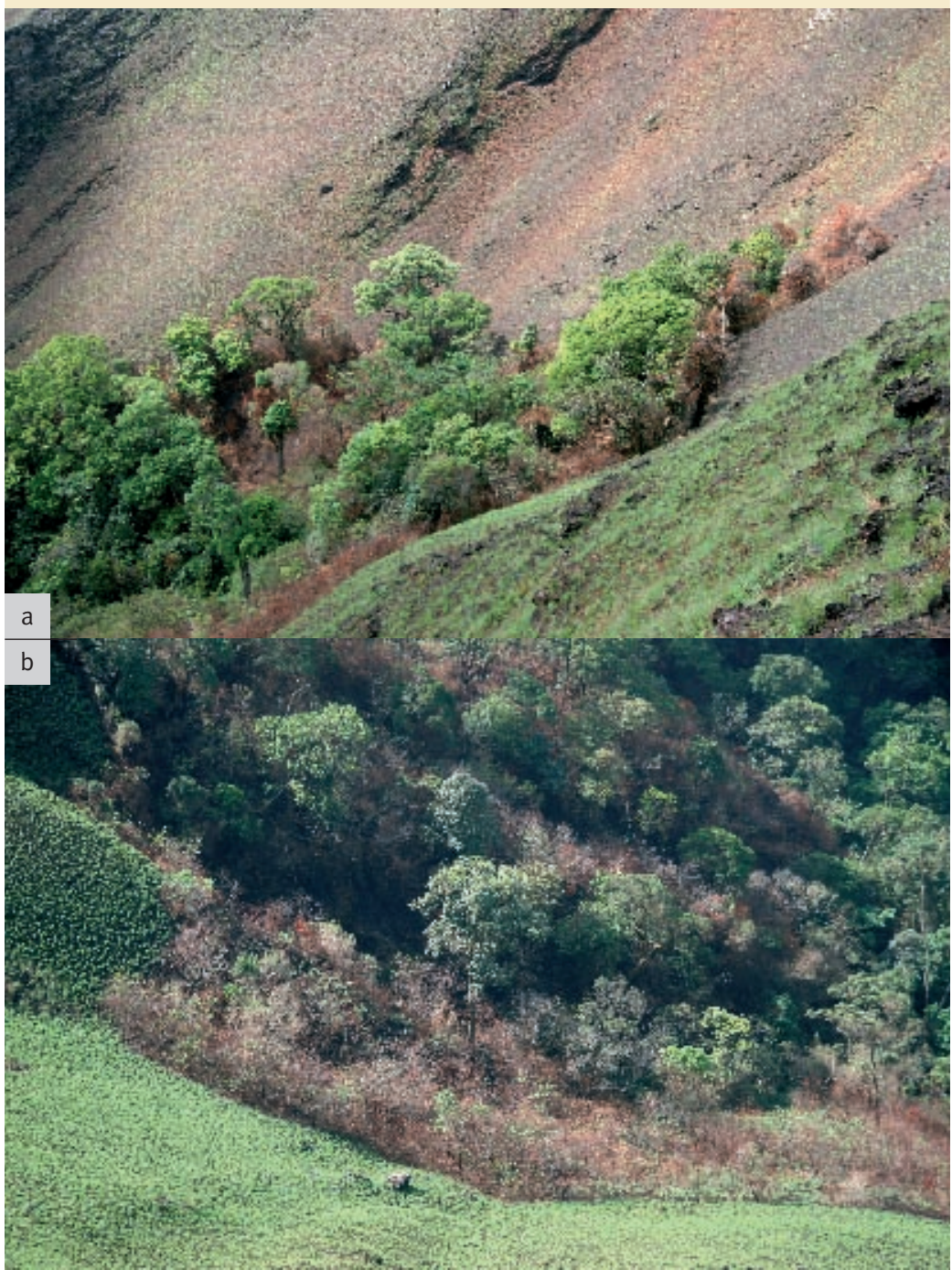

Photos 12

$\mathrm{a}$ et $\mathrm{b}$. Forêts de ravin dégradées par le feu avec altération des lisières et pénétration du feu dans le sous-bois de la forêt. Photo P. Poilecot.

\section{Impact du feu sur les vertébrés terrestres}

L'impact majeur du feu sur les vertébrés terrestres concerne les changements dans les habitats avec une diminution des abris et une augmentation de la prédation. Dans les savanes guinéennes de Côte d'Ivoire, l'absence de feu n'a pas d'effet sur la diversité spécifique en amphibiens et reptiles mais influe sur leur densité, celle-ci étant plus forte dans les savanes non périodiquement brûlées. Des études conduites en Australie montrent que le type de feu a peu d'incidence sur la richesse spé- cifique des savanes. Malgré quelques exceptions ou variations locales, la plupart des espèces ont une forte résilience au feu.

Au cours du Biopa, les captures de petits mammifères ont montré que certaines espèces avaient migré des savanes à Loudetia kagerensis vers des formations buissonnantes proches, après le passage des feux.

Ces perturbations ne sont que temporaires et les animaux réinvestissent leurs territoires au fur et à mesure de la reconstitution du couvert herbacé. Les conséquences du feu affectent les ressources alimentaires, devenant rapidement localement inexistantes ou réduites après les incendies. Malgré cela, comme pour les végétaux, la recoIonisation du milieu par la faune est relativement rapide. Le feu « rajeunit » temporairement les physionomies végétales en chassant certains occupants mais en permettant d'en accueillir d'autres après l'ouverture du milieu. Des oiseaux comme l'alouette à nuque rousse (Mirafra africana henrici), le pipit à long bec (Anthus similis bannermani) et le traquet pâtre (Saxicola torquata nebularum) profitent certainement du passage des feux. Il en est de même pour les rapaces pour lesquels la chasse est plus facile. La reconquête des espaces se fait souvent de façon " endogène », par les espèces présentes sur place avant le feu. Le passage des feux est également bénéfique pour les herbivores (sauvages et domestiques), du fait d'un regain de pâturage en saison sèche. La densité des mammifères sauvages est très faible dans cette partie du Nimba. Seules quelques observations de céphalophes (Cephalophus monticola maxwelli), de traces et fèces de guib harnaché (Tragelaphus scriptus) et de buffle (Syncerus caffer nanus) confirment la présence de ces espèces, même dans les savanes submontagnardes. Le daman des rochers (Procavia capensis), inféodé aux milieux rocheux et herbacés, est encore bien représenté dans ce massif.

\section{Impact du feu sur les invertébrés terrestres}

Au sein des savanes tropicales, les insectes et autres arthropodes peuvent être considérés comme des " ingénieurs " des écosystèmes et constituent des bioindicateurs clés de la santé des écosystèmes (ANDERSEN et al., 2003). Le meilleur exemple est fourni par les termites et les fourmis qui agissent sur la structure et la texture du sol, sa porosité, sa fertilité et la minéralisation des nutriments. Dans certaines savanes d'Australie, il existe une corrélation positive significative entre la densité des termites et celle des oiseaux, mammifères et reptiles insectivores. 
Le feu intervient directement sur les invertébrés en les tuant ou les obligeant à migrer du fait d'une perturbation de l'habitat et d'une diminution brutale des ressources alimentaires. D’une façon générale, la réponse des espèces à un régime de feux extrême (feux tardifs ou absence de feu) est assez limitée. Ces animaux possèdent une forte résilience permettant une reconstitution rapide des populations d'origine, tant au niveau de l'espèce que des familles.

Dans les savanes à rôniers (Borassus aethiopium) du centre de la Côte d'Ivoire, les feux conduisent à une diminution d'environ $39 \%$ des arthropodes mais les populations commencent à se rétablir quelques mois après le passage des incendies (LAMOTTE, 1981). La dynamique des populations est également régie, pour de nombreux groupes, par d'autres facteurs comme la pluviométrie. Si le type de feu ne semble pas avoir un grand impact sur la faune des savanes, la fréquence des incendies est plus importante à prendre en considération.

\section{Problématique}

Les forêts denses jouent un rôle socio-économique important pour les populations en leur fournissant les produits de première nécessité. Mais de nombreuses contraintes (faiblesse institutionnelle, besoins immédiats des communautés, superposition des droits coutumier, religieux et moderne sur la terre, etc.) pèsent sur leur gestion. Dans la région des monts Nimba, les forêts planitiaires et de piémont sont les plus menacées du fait des défrichements et des feux culturaux (photo 14).

Ces derniers ont grandement contribué à la transformation de cet écosystème et représentent un problème majeur pour le maintien du capital forestier et de la biodiversité. En Guinée forestière, la déforestation touche environ 35000 ha de forêt par an, soit un taux de recul moyen des forêts proche de $5 \%$.
Les « brousses » herbacées ou arbustives, qui constituent un stade évolutif vers la forêt primitive, sont souvent la proie des flammes et demeurent ainsi à l'état de clairières.

Dans les savanes soudano-guinéennes et guinéennes, l'arrêt de la pratique des feux conduit à une évolution de la végétation vers la forêt dense, lorsque les conditions édaphiques le permettent. Les espèces savanicoles pyrophytes sont progressivement remplacées par des plantes forestières, plus sensibles au feu, mais plus compétitives que celles de la savane en l'absence de feu. Le tapis graminéen pyrophyte des savanes est ainsi induit par les feux périodiques. D'une façon générale, les incendies tardifs sont plus agressifs vis-à-vis des arbres et arbustes et leur régénération, même pour ceux considérés comme " pyrotolérants ». Le passage d'un feu en mars-avril détruit la nouvelle feuillaison des ligneux, obligeant ainsi les individus à la renouveler en puisant dans leurs réserves.

Les feux façonnent la structure démographique du peuplement ligneux. Dans une savane non soumise au pâturage, les incendies, les arbres et les plantes herbacées, par le jeu de la compétition, limitent le développement des jeunes ligneux et la population reste stable. La densité des ligneux dépend alors des conditions pédologiques et en particulier de la fertilité.

Il y a donc nécessité de trouver un compromis entre le développement de l'élevage et le maintien de pâturages pouvant supporter une charge de bétail croissante. Cela avait été souligné par RENARD et al. (1981) au sujet des savanes périforestières de la vallée de la Gouan. Le pâturage, de plus en plus intensif dans les savanes de piémont, est un risque plus important que celui des feux dans le maintien de l'originalité de ces formations. Certaines d'entre elles, mises en culture de façon temporaire, ont évolué vers des formations ligneuses.

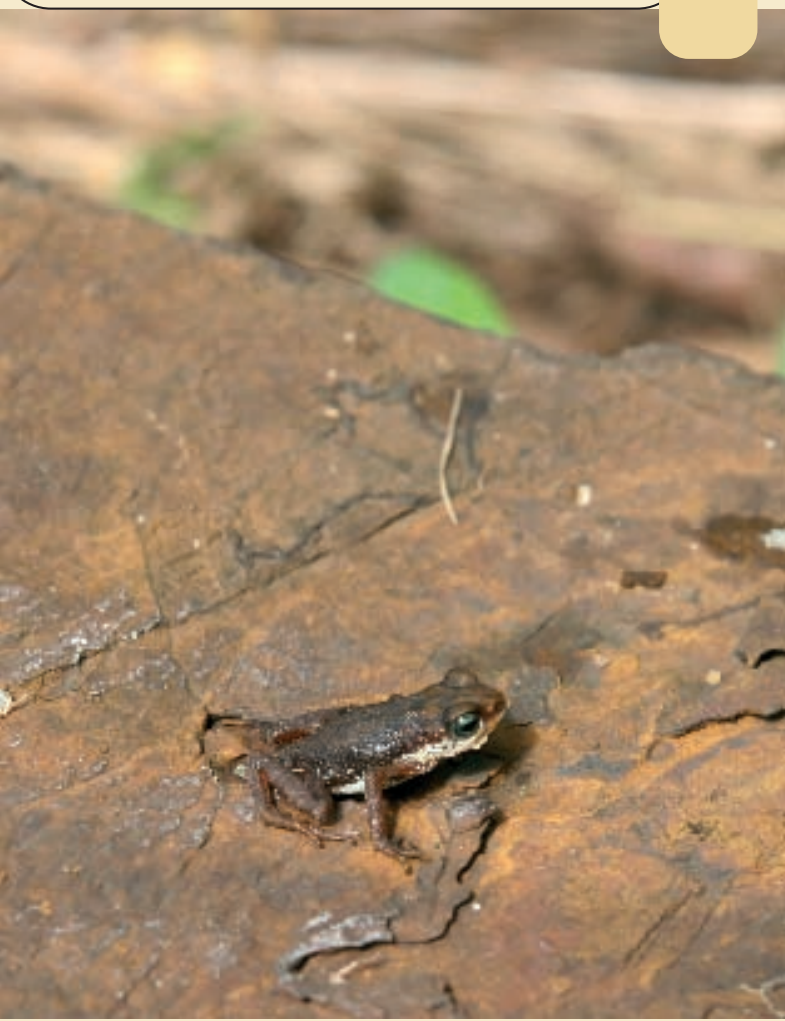

Photo 13.

Crapaud vivipare, Nectophrynoides occidentalis, à 1670 m d'altitude. Photo P. Poilecot.

L'absence de bétail et de grands mammifères dans les savanes d'altitude pourrait laisser penser que le problème est plus simple à considérer. Malgré des sols superficiels, le milieu submontagnard permet le développement d'une végétation herbacée dense. Les travaux de FournIER (1987), sur la biomasse des savanes à Loudetia kagerensis, montrent que les phytomasses maximales des crêtes du Nimba sont particulièrement élevées. La repousse du tapis herbacé, trente jours après le passage du feu, donne une production comprise entre 25 et 50 $\mathrm{kg} / \mathrm{ha} /$ jour, proche de celle obtenue dans les savanes préforestières (guinéennes) de Côte d'Ivoire (CÉSAR, 1971 ; AbBadie, 1983). Ces caractéristiques sont celles de savanes humides "éniautopyres ". Les récents inventaires floristiques conduits au cours de la saison des pluies (POILECOT et al., 2008) ont mis en évidence la riche diversité floristique de ces savanes qui renferment près de quarante espèces de Poaceae. On pourrait les qualifier de 
« vraies » savanes, en opposition aux savanes des zones plus sèches caractérisées par une forte proportion d'annuelles et dans lesquelles l'impact du feu est totalement différent, souvent préjudiciable à la végétation.

L'absence de feux risque de conduire à une érosion de la diversité floristique en freinant la régénération et le développement de certaines espèces. Elle peut également entraîner un embroussaillement local des savanes montagnardes par des ligneux très agressifs comme Kotschya ochreata, Harungana madagascariensis ou Trema orientalis. La présence d'herbacées rudérales (Ageratum conyzoides, Emilia coccinea, Paspalum scrobiculatum), témoins d'une perturbation du milieu, n'est pas inquiétante car ce sont des annuelles ayant un impact négligeable.

Le développement de Melinis minutiflora qui, localement, se substitue à Loudetia kagerensis, même à haute altitude (1 $600 \mathrm{~m})$, est beaucoup plus problématique. L'invasion de cette espèce avait été constatée voilà une cinquantaine d'années, même dans les zones non perturbées par la prospection minière. Cette graminée, introduite au Brésil comme plante fourragère, est maintenant considérée comme une peste dans les cerrados qui ont été protégés des feux (MISTRY, BERARDI, 2006).

Bien que les grands mammifères soient peu représentés dans les savanes du Nimba, il n'est pas utopique de songer à ce que les populations animales se reconstituent, petit à petit, si la surveillance de la réserve contre le braconnage est efficace et durable. L'intérêt de maintenir des zones de pâturage en altitude doit donc faire partie de l'aménagement du site et de la gestion des feux.

Au regard des conséquences du feu sur la végétation et la faune et dans le contexte socio-économique actuel qui prévaut dans la région des monts Nimba, en particulier en matière d'élevage, comment aborder la question des feux de brousse dans un souci de production et de conservation de la biodiversité ?

\section{Le feu comme un outil de gestion}

Jusqu'en 1994, la suppression des feux au Mali a entraîné la recrudescence de feux tardifs extrêmement dommageables à la végétation et à la faune, entraînant parfois la destruction de villages. Le retour à la pratique des feux a été souhaité par les populations rurales pour maintenir une mosaïque de paysages indispensable à leur système de production (MISTRY, BERARDI, 2006 ; LARIS, BAKKOURY, 2008). Diverses études, sous différents climats, ont montré l'intérêt des feux, à des périodicités plus ou moins grandes, pour le maintien ou l'accroissement de la richesse et de la diversité floristiques. Le feu agit sur la productivité des savanes et la teneur en protéines dans des graminées en épiaison est supérieure à celle observée chez les espèces au sein de savanes protégées des feux pendant plusieurs années. Comme le soulignent MBo et al. (2002), la lutte active contre le feu a montré ses limites quant à la mobilisation de moyens humains et logistiques importants. Les populations locales sont souvent exclues dans les pratiques de lutte, les budgets sont insuffisants, les moyens non pérennisés et la sensibilisation très souvent reléguée au second plan. La mise en place de pare-feux (nus ou enherbés) et leur entretien, qu'il soit manuel ou mécanisé, sont coûteux et d'une efficacité relative face à la violence du feu, l'intensité du vent et l'abondance du matériel végétal qui brûle. Un réseau bien étudié de pare-feux s'avérera totalement inefficace dans des régions soumises au braconnage.

Dans les monts Nimba, la lutte contre les feux pose, de plus, le problème de l'accès aux savanes d'altitude.

Les savanes tropicales sont la proie des flammes, d'année en année, malgré de nombreux projets de développement et dispositifs mis en place dans le but de les limiter. Les différents acteurs occupant ces savanes ou les forêts qui les bordent ont des intérêts communs ou divergents dans la gestion de l'environnement et des ressources naturelles. Les conflits agriculteurs/éleveurs ou forestiers/éleveurs sont là pour le rappeler au quotidien. Les «prairies » d'altitude ne sont pas soumises au pâturage du bétail, supportent une très faible densité d'herbivores sauvages et ne sont pas l'objet de fréquentation touristique. Alors, pourquoi soumettre cet écosystème au feu ? Des feux parfaitement gérés dans les savanes de basse altitude pourraient éviter les incendies des crêtes ayant un impact sur les formations forestières. La gestion de tels feux ne serait pas sans faille avec le risque d'incendies tardifs couvrant de plus grandes superficies et dommageables à la régénération ligneuse, aux massifs forestiers ainsi qu'aux sols car favorisant le ruissellement.

Le feu peut être considéré comme un fléau lorsqu'il n'est pas maîtrisé. Une politique de feux précoces est un moindre mal si l'on ne dispose pas des moyens nécessaires (humains et financiers) pour mener à bien une stratégie de gestion des incendies.

Cette pratique est certainement la plus simple à mettre en œuvre en garantissant le maintien en équilibre des savanes, de leur diversité floristique et la réussite des activités liées à l'élevage sans nuire à la composition ni à la structure des formations herbeuses.

La protection intégrale des savanes submontagnardes contre le feu, avec la mise en place de parefeux nus (dégagement de la matière herbacée sèche, feux précoces), pourrait être envisagée sur des sites de superficie limitée et faciles à surveiller, dans le cadre d'un dispositif expérimental avec l'objectif de :

- comparer la dynamique de la végétation herbacée entre des parcelles protégées du feu et d'autres soumises à différents rythmes d'incendies (précoces et tardifs) ;

- comparer la richesse et la diversité floristiques des milieux brûlés et protégés ;

- suivre la dynamique de Melinis minutiflora ;

- suivre la dynamique des populations de vertébrés et d'invertébrés. 
Les conséquences des feux de brousse doivent être envisagées et analysées de façon objective en prenant en compte le facteur temps. Pour mesurer l'impact du feu sur le milieu, la collecte d'informations est nécessaire dès le passage des incendies, tant sur la végétation que sur la faune : premiers stades de colonisation, succession des espèces, proportion pérennes/annuelles, stratification des peuplements, comparaison des milieux et des populations reconstitués avec ceux et celles d'origine, etc. Des expérimentations sur le long terme, en milieu méditerranéen, ont montré que ce sont les espèces qui existaient avant le feu qui se réinstallent et reconstituent les communautés selon un processus d' "autosuccession » plutôt que de « remplacement » (Trabaud, 2008).

Les incendies sont indispensables au maintien de la structure de la végétation, de la strate graminéenne et du cortège floristique de la savane et agissent donc plus comme un facteur de conservation de l'équilibre biologique que comme un agent perturbateur ou appauvrissant de cet écosystème. Cette constatation s'applique aussi bien dans les savanes de piémont que dans celles d'altitude. La plupart des herbacées qui réapparaissent après le passage du feu sont des plantes vivaces, tolérantes ou "résilientes " au feu, avec certainement, en complément, un apport de graines qui permet le développement d'annuelles.

Les feux de brousse sont considérés comme un facteur écologique par les naturalistes ou les conversationnistes mais aussi comme un facteur anthropique par les chercheurs des sciences sociales. Ils se placent ainsi à l'interface nature/société (DoLIDON, 2005) et constituent un phénomène complexe qu'il est indispensable d'aborder à des échelles spatiales et temporelles différentes. Au sein des terroirs, les facteurs déterminants des feux sont en partie les mêmes que ceux d'un territoire mais avec, en complément, des paramètres locaux liés aux conditions édaphiques et aux systèmes de production. On peut ainsi considérer que les feux de brousse maintiennent une mosaïque de phytocénoses, adaptées à divers bouleversements occasionnés par les actions humaines passées (défrichements, jachères, feux divers, etc.), dans un état d'équilibre « métastable », tant que les conditions environnementales le permettent.

\section{Des partenariats indispensables}

Dans le cadre de la réserve des monts Nimba, mais également des aires protégées en général, la réussite de la mise en œuvre d'une " gestion » des feux de brousse dépend avant tout de la volonté des gestionnaires de s'impliquer dans la stratégie définie et dans la surveillance du site. Ces derniers doivent initier des collaborations et partenariats avec toutes les parties intéressées (agences de financement, projets de développement, communautés locales, autorités administratives et politiques, instituts de recherche), en prenant en compte les souhaits et besoins des populations riveraines. Un travail de communication est alors indispensable, concernant aussi bien les feux culturaux que ceux qui parcourent les savanes. L'intensification de l'agriculture itinérante, due à une population de plus en plus dense, conduit à des défrichements considérables et à la disparition du patrimoine forestier du pays. La composante "surveillance» de la réserve est un élément clé de la réussite des activités de conservation, non seulement en Guinée mais dans chacun des pays qui abritent le massif des monts Nimba. Tous les efforts déployés pour maintenir en défens des zones protégées ou destinées à la recherche seront anéantis par le seul passage d'un feu dû à un acte malveillant ou à des braconniers. L'intégrité de la végétation et de la faune de ces montagnes ne peut être préservée sans la mise en œuvre d'une action commune régionale considérant la réserve comme une aire protégée transfrontalière.

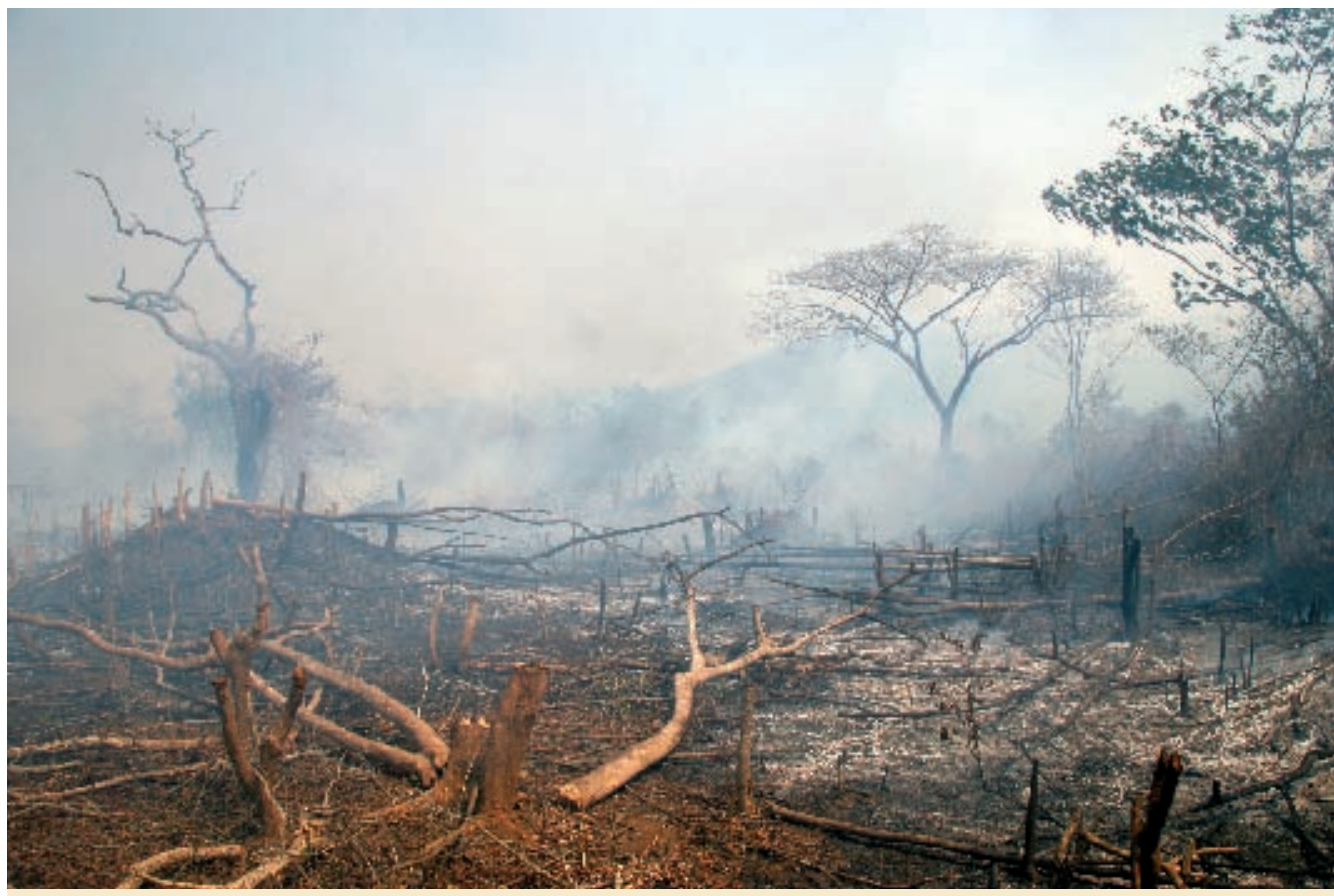

Photo 14.

Feu de défrichement dans la zone de piémont de la réserve des monts Nimba. Photo P. Poilecot. 


\section{Références bibliographiques}

ABBADIE L., 1983. Contribution à l'étude de la production primaire et du cycle de l'azote dans les savanes de Lamto (Côte d'Ivoire). Travaux des Chercheurs de la Station de Lamto (Côte d'Ivoire), n 1,135 p.

ANDERSEN A. N., ORGEAS J., BLANCHE R. D., LOWE L. M., 2003. Terrestrial insects. In : Fire in Tropical Savannas : the Kapalga Experiment. New York, États-Unis, Springer, 107-125.

ARBONNIER M., 2008. Faut-il tolérer les feux dans les parcs nationaux d'altitude en Afrique centrale. Bois et Forêts des Tropiques, 296 (2) : 39-43.

BOWMAN D. M. J. S., WILSON B. A., 1995. Munmarlary revisited : response of a north Australian Eucalyptus tetrodonta savanna protected from fire for 20 years. Australian Journal of Ecology, 20 (4) : 526-531.

CÉSAR J., 1971. Étude quantitative de la strate herbacée de la savane de Lamto (Moyenne Côte d'Ivoire). Thèse de doctorat de $3^{\text {e }}$ cycle, Faculté des Sciences, Paris, 195 p.

DOLIDON H., 2005. L'espace des feux en Afrique de l'Ouest. L'analyse spatio-temporelle d'un phénomène d'interface nature/société. Thèse de doctorat, Université de Caen/BasseNormandie, $414 \mathrm{p}$.

DOUGLAS M. M., TOWNSED S. A., LAKE P. S., 2003. Streams. In : Fire in Tropical Savannas : the Kapalga Experiment. New York, États-Unis, Springer, 59-78.

FOURNIER A., 1987. Quelques données quantitatives sur les formations herbacées d'altitude des monts Nimba (Ouest africain). Bulletin du Muséum National d'Histoire Naturelle, Paris, $4^{\mathrm{e}}$ série, 9 , section $B$, Adansonia, $n^{\circ} 2$ : 153-166.

JACQUES-FÉLIX H., 1987. Le Melinis minutiflora est une graminée africaine. Bulletin du Muséum National d'Histoire Naturelle, Paris, $4^{\text {e }}$ série, 9 , section $B$, Adansonia, $n^{\circ} 4$ : 461-462.
KOECHLIN J., 1998. État actuel de la végétation du Nimba. In : Le Mont Nimba : réserve de biosphère et site du patrimoine mondial (Guinée et Côte d'Ivoire). Initiation à la géomorphologie et à la biogéographie. Paris, France, Unesco/Mab/Pnud, 77-80.

LAMOTTE M., 1981. Structure et fonctionnement des écosystèmes de la savane de Lamto (Côte d'Ivoire). In : Unesco, Recherches sur les ressources naturelles. XVI. Écosystèmes pâturés tropicaux. Unesco, Presses Universitaires de France, 529-580.

LAMOTTE M., 1998. Le climat du Nimba. In : Le Mont Nimba : réserve de biosphère et site du patrimoine mondial (Guinée et Côte d'Ivoire). Initiation à la géomorphologie et à la biogéographie. Paris, France, Unesco/ Mab/Pnud, 37-54.

LAMOTTE M., ROUGERIE G., 1998. Les traits principaux de la géologie et de la géomorphologie du Nimba. In : Le Mont Nimba : réserve de biosphère et site du patrimoine mondial (Guinée et Côte d'Ivoire). Initiation à la géomorphologie et à la biogéographie. Paris, France, Unesco/Mab/Pnud, 13-36.

LAMOTTE M., ROY R. (éd.), 2003. Le peuplement animal du Mont Nimba (Guinée, Côte d'Ivoire, Liberia). Paris, France, Muséum national d'histoire naturelle, coll. Mémoires, 724 p., 1 cédérom.

LARIS P., BAKKOURI A., 2008. Nouvelles leçons d'une vielle pratique : mosaïque du feu dans les savanes du Mali. Bois et Forêts des Tropiques, 296 (2) : 5-16.

LAUGINIE F., 2008. Conservation de la nature et aires protégées en Côte d'Ivoire. Abidjan, Côte d'Ivoire, Ceda/Nei, 668 p.

MBO C., GOUDIABY A., SAMBOU B., BA A. T., 2002. Towards a community forest strategy to minimise the occurrence of bushfires in Senegal. Serein Occasional Paper $n^{\circ} 13: 137-146$.
MISTRY J., BERARDI A., 2006. Savanna and dry forests : Linking People with Nature. Aldershot, Royaume-Uni, Ashgate.

POILECOT P., 2008. Gestion des feux de brousse sur le versant guinéen des Monts Nimba : Rapport de mission. Cirad/Afnat, 25 p.

POILECOT P., HABA O. O., BILIVOGUI D., 2008. Contribution à l'étude de la composition du tapis graminéen des savanes d'altitude sur le versant guinéen du Mont Nimba. Smfg/Cirad, 55 p. RENARD J. F., COMPÈRE R., MATHIEU L., ALLIOU T. D., 1981. Les savanes périforestières de la vallée de la Gouan en République de Guinée. I. Quelques aspects de la relation solsplantes. Bulletin des Recherches Agronomiques de Gembloux, 16 (4) : 379-398.

SCHNELL R., 1952. Végétation et flore de la région montagneuse du Nimba. Dakar, Sénégal, Ifan, Mémoire $n^{\circ} 22$, $604 \mathrm{p}$.

SCHNELL R., 1987. Les formations herbeuses montagnardes des monts Nimba (Ouest africain). Bulletin du Muséum National d'Histoire Naturelle, $4^{\mathrm{e}}$ série, 9 , section $B$, Adansonia, $n^{\circ} 2: 137-151$.

SCHNELL R., 1998. Le Mont Nimba, carrefour ouest-africain des flores. In : Lamotte M. (éd.). Le Mont Nimba : réserve de biosphère et site du patrimoine mondial (Guinée et Côte d'Ivoire). Initiation à la géomorphologie et à la biogéographie. Paris, France, Unesco/Mab/Pnud, 55-76.

TRABAUD L., 2008. La réponse de la végétation aux incendies. In : Le feu dans la nature : mythes et réalité. Toulouse, France, Éditions Plume de carotte, coll. Écologistes de l'Euzière, 51-70.

YEATON R. I., FROST S., FROST P. G. H., 1986. A direct gradient analysis of grasses in a savanna. Africa Journal of Science, 82 : 482-486. 